\title{
Was wäre, wenn ...
}

\section{Christoph Bosshard}

Dr. med., Vizepräsident der FMH, Departementsverantwortlicher Daten, Demographie und Qualität DDQ

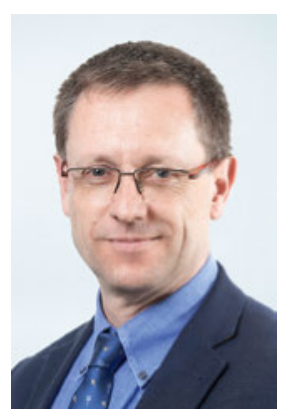

... der Stimmbürger dieses Gesundheitswesen in dieser hochstehenden und rasch erreichbaren Form genau so will? Etwa mit diesen Worten beendete Jonas Projer die Arena-Sendung vom 20. April 2018 unter dem Titel "Gesundheitssystem auf der Intensivstation». Bezeichnenderweise sassen dabei die Vertretungen der Patientinnen und Patienten sowie der Ärztinnen und Ärzte nur in der zweiten Reihe und durften mehrheitlich dabei zusehen, wie von Seiten Politik und Kostenträger Positionen abgetauscht wurden. Alleine von Kosten war die Rede, nicht jedoch von der Finanzierung oder gar vom Mehrwert - vom Nutzen. Und hier sind wir bei der alten Wahrheit: Wer nur den Preis kennt, hat vom Wert keine Ahnung. Welchen wachsenden Nutzen unsere Gesundheitsausgaben für Patienten und Volkswirtschaft bringen, zeigten bereits viele Beiträge in der SÄZ [1]. Dass das KVG den Nutzen nicht abbildet, ist seiner Konstruktion mit Beschränkung auf die Kostenvergütung von Heilkosten geschuldet - im Gegensatz zum UVG, welches auch für Taggelder und Renten zuständig ist. Wenn ich dann noch Diskussionen höre, ob ab einem Alter von 90 Jahren eine Hüftgelenksersatz-Operation noch zu Lasten der Solidarversicherung zu bezahlen sei, realisiere ich vollends, dass komplett ausgeblendet wird, dass bereits ab einem halben Jahr verzögertem Pflegeheimeintritt der

Würde die Politik die Bedürfnisse der Patienten ins Zentrum stellen, hätten wir weniger Bürokratie und geringere Kosten.

Hüftgelenksersatz auch finanziell rentiert [2]. Und mit Verlaub: Das Alter alleine ist nun wirklich kein genügend gut selektionierender Indikator.

Weitere Ideen umfassen die Erweiterung der Kostenbeteiligung durch die Versicherten mit exorbitanten Franchisen, dies bei weltweit höchstem Out-of-PocketAnteil der Gesundheitskostenfinanzierung in unserem Land. Gleichzeitig verzeichnen die Krankenkassenprämien ein deutlich stärkeres Wachstum als die Kosten unseres Gesundheitswesens. Denn die Kosten steigen seit Jahren linear, dies notabene trotz eines deutlich überlinearen Zuwachses der Altersklasse 75+ mit einem dreifach erhöhten Bedarf an Leistungen. Gerade dieser Umstand ist Ausdruck einer kontinuierlichen Effizienzsteigerung.

Nun erhofft sich die Politik Kostensenkungen durch einen Gesetzesvorschlag im KVG mit dem Titel «Stärkung von Qualität und Wirtschaftlichkeit (15.083)». Auffällig ist immer wieder, dass einfache Patentrezepte zu kurz greifen. Sowohl Patientinnen und Patienten wie auch deren Ärztinnen und Ärzte und Gesundheitsfachpersonen bilden das Zentrum des Gesundheitswesens.

\section{Nur von Kosten ist die Rede, nicht aber vom}

Nutzen medizinischer Leistungen: Wer nur den Preis kennt, hat vom Wert keine Ahnung.

Sie an den Rand zu drängen ist weder in einer ArenaSendung noch im politischen Gesetzgebungsprozess zielführend. Deshalb ist das Parlament gut beraten, auf bewährte Strukturen aufzubauen oder von deren Erfolgsrezept zu lernen: Die Stiftung Patientensicherheit Schweiz, der ANQ oder auch QualiCCare sind erfolgreiche Organisationen, welche sich mitunter dadurch auszeichnen, dass die Verbände ihre Vertretung in die strategischen Gremien delegieren und Bottom-up das fachliche kollektive Wissen einbinden. So ist das Commitment der Leistungserbringer deutlich besser abzuholen als in neu zu schaffenden Strukturen. Qualität ist in erster Linie einer Haltung geschuldet, einer intrinsischen Motivation, welche seit jeher Bestandteil des ärztlichen Handelns war und ist.

Was wäre, wenn ... die Politik mit dem neuen Gesetz die Rahmenbedingungen und die Finanzierung der Qualitätsarbeit so regeln würde, dass wirklich die Bedürfnisse der Patientinnen und Patienten im Zentrum stünden und die qualitativ hochstehende Arbeit der Leistungserbringer in würdigender Art und Weise unterstützt würde? Dann nämlich hätten wir weniger Bürokratie, weniger sinnlosen administrativen Aufwand, weniger gesetzliche Fallstricke, geringere Kosten, ABER wieder mehr Zeit, um uns den wirklich wichtigen Inhalten, nämlich unseren Patientinnen und Patienten, zu widmen. 